\title{
Recent Results from the IceCube Neutrino Observatory
}

\section{Chad Finley* for the IceCube Collaboration}

Stockholm University

E-mail: cfinley@fysik.su.se

\begin{abstract}
Observations spanning $\mathrm{TeV}$ gamma rays to $\mathrm{EeV}$ cosmic rays suggest that a correlated flux of neutrinos within this energy range should also exist. Hadronic acceleration should lead to pion production via $\mathrm{p}-\mathrm{p}$ and $\mathrm{p}-\gamma$ interactions, and the subsequent decay of charged pions leads to neutrinos. The principal mission of the IceCube Neutrino Observatory is to detect these high energy neutrinos and identify their sources. Leading source candidates are objects long suspected of accelerating cosmic rays, such as supernova remnants, microquasars, active galactic nuclei, and gamma ray bursts. In addition to the expected conventional astrophysical sources, a wide range of dark matter models predict that neutrinos in the $\mathrm{GeV}$ to $\mathrm{TeV}$ range may be detected from the Sun due to the annihilation of dark matter particles, which would accumulate in its center.

Located at the geographic South Pole, IceCube has been under construction since 2005 and is scheduled to be completed in 2011. IceCube is instrumenting one cubic kilometer of the clear Antarctic ice sheet with an array of over 5000 modules to detect the Cherenkov light from relativistic particles traversing the ice. High energy neutrinos which interact within or near the detector lead to daughter particles such as muons whose km-long tracks can be reconstructed and used to infer the incoming neutrino direction with as little as $0.5^{\circ}$ uncertainty for high energy events. While IceCube cannot claim detection of extraterrestrial neutrinos yet, tens of thousands of high energy neutrinos from cosmic ray air showers have already been observed. During the same time, billions of cosmic ray showers have been observed in the form of muons passing downward through the in-ice detector. This high statistics sample has revealed the existence in the southern sky of a large scale anisotropy of cosmic rays above $\mathrm{TeV}$ energies, similar to the anisotropy observed in the northern sky.
\end{abstract}

Results of recent searches for neutrino point sources, indirect dark matter detection, and cosmic ray anisotropies will be presented.

35th International Conference of High Energy Physics

July 22-28, 2010

Paris, France

${ }^{*}$ Speaker. 


\section{Introduction}

The IceCube Neutrino Observatory at the geographic South Pole, under construction since 2005 , is scheduled to be completed in 2011. Its principal mission is to detect high energy neutrinos from astrophysical sources, opening a window onto astronomy that complements the familiar electromagnetic one. The small neutrino cross-sections necessitate a large detector volume. The IceCube detector comprises 5160 digital optical modules (DOMs) deployed in a three-dimensional array approximately one cubic-kilometer in size and centered $2 \mathrm{~km}$ deep in the clear Antarctic ice. Each DOM consists of a photo-multiplier tube and electronics for digitization of waveforms and communication with neighboring DOMs and the surface. Cherenkov light from the passage of a relativistic charged particle through the ice creates a pattern of "hit" DOMs in the array, and the position and timing of the hits is used to reconstruct the path of the particle. The vast majority of these particles are muons, arriving from cosmic ray air showers occurring in the atmosphere above the site and triggering the detector at an approximate rate of $2 \mathrm{kHz}$ in the current configuration. Cosmic-ray air showers also produce neutrinos plentifully. Though most go undetected, roughly one out of $10^{6}$ triggered events is due to a neutrino interaction in the ice or bedrock below the detector that results in a muon. The simplest way to positively identify such neutrino-induced muon tracks is to select only the up-going tracks, using the Earth to filter out all other particles. While more sophisticated neutrino event selections are under development, IceCube already detects $\sim 100$ neutrinos per day in this way.

The current (final) IceCube array consists of 79 (86) strings lowered into the ice, each equipped with 60 DOMs. Here, results are presented from completed analyses of the 22-string season (2007 May 31 to 2008 April 4) and the 40-string season (2008 April 5 to 2009 May 20) data.

\section{Neutrino Point Source Search}

The most sensitive search to date for point sources of high energy ( $>\mathrm{TeV}$ ) neutrinos has been performed using the data from the 40-string IceCube configuration [1]. The final event selection consists of 36900 events over the sky, consistent with the number of atmospheric muons and neutrinos expected from background cosmic rays. An unbinned maximum likelihood analysis was performed, fitting for the excess number of events and their spectral index at each position in the sky. The results are shown in Fig. 1. For each direction, the plot shows the significance (log of the estimated p-value) of the best-fit signal parameters. Note that the analysis includes not only the northern sky (corresponding to upgoing neutrino events), but the southern sky as well. Because of the much larger background of down-going cosmic ray muons, the neutrino point source sensitivity in this analysis of the southern sky is limited to energies above $1 \mathrm{PeV}$, where a harder $E^{-2}$ spectrum from a neutrino point source can stand out against the softer $E^{-2.7}$ cosmic ray spectrum.

The most significant spot in the all-sky search in Fig. 1 is at $113.75^{\circ}$ r.a., $15.15^{\circ}$ dec. Considered as an isolated analysis, the p-value for this spot is $5.2 \times 10^{-6}$. However, this is a typical result for the all-sky search: performing the same analysis on time-scrambled data yields a similar or smaller p-value somewhere in the sky about $18 \%$ of the time. The analysis is also performed on a very restricted list of directions corresponding to 39 promising source candidates selected $a$ priori. None of the source directions on this list yielded a significant excess. 


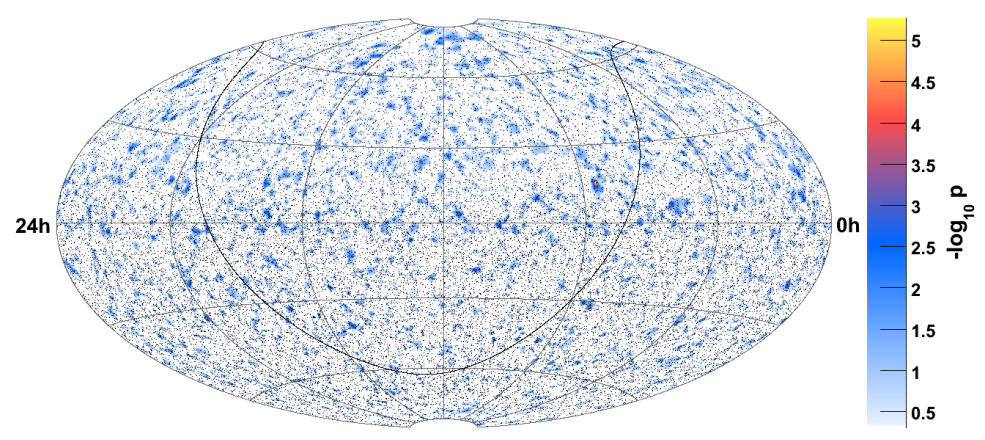

Figure 1: Skymap in equatorial coordinates of the significance (minus the logarithm of the estimated pvalue) at each direction in the sky for the best-fit result of a maximum likelihood analysis looking for an excess of signal-like events. The northern sky is predominantly atmospheric neutrino-induced muons; the southern sky predominantly cosmic ray muons. Points are the reconstructed event locations. The solid black curve is the galactic plane.

\section{Indirect Search for Dark Matter}

An important potential source of astrophysical neutrinos is the annihilation of dark matter particles. For example, in models where dark matter consists of Weakly Interacting Massive Particles (WIMPs), these particles are expected to occasionally scatter off of nuclei in the Sun, get captured in the gravitational potential, and sink toward the solar center after further scatterings. WIMP-WIMP annihilations will occur, leading to $\mathrm{GeV}$ to $\mathrm{TeV}$ neutrinos via many channels, each with a characteristic neutrino energy spectrum. As the density of dark matter particles grows at the center, the rate of WIMP-WIMP annihilation will increase until it equals the capture rate. For this reason, indirect dark matter searches using neutrino signals from the Sun are sensitive to the WIMP-nucleon scattering cross-section.

Fig. 2 shows, as a function of mass, the spin-dependent cross section limits and predicted sensitivities for a number of current and future experiments. The IceCube limits shown were obtained using data from the 22-string detector configuration (above $200 \mathrm{GeV}$, [2]) and the predecessor AMANDA experiment (below $200 \mathrm{GeV}$, [6]). Because a denser spacing of optical modules (as in AMANDA) lowers the energy threshold, the completed IceCube will include six special strings in the center of the array with the DOMs concentrated at the bottom, known as the DeepCore sub-detector.

\section{Cosmic Ray Anisotropy}

The cosmic rays which trigger the in-ice detector are a background for neutrino searches, and they are difficult to do pointing astronomy with, because their paths are strongly bent by galactic magnetic fields. With high statistics, however, it is possible to observe small amplitude deviations from a completely isotropic distribution of cosmic ray arrival directions. Fig. 3 shows the first such observation of cosmic ray anisotropy in the southern sky. This is based on an analysis of 4 billion down-going cosmic ray muon events recorded by the IceCube detector in the 22-string 


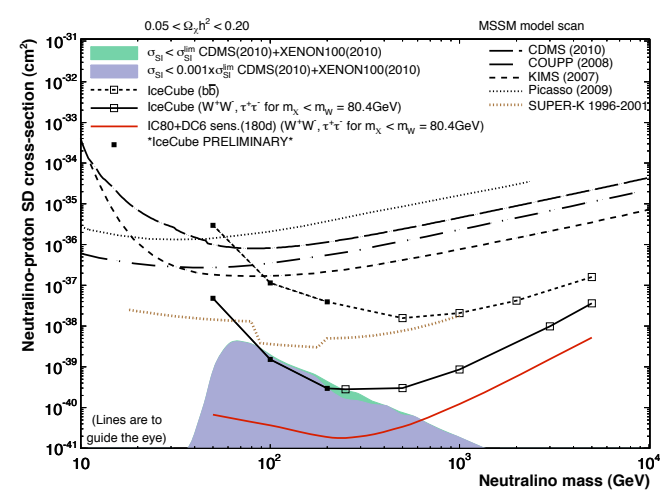

Figure 2: 90\% CL upper limits and sensitivities to the spin-dependent WIMP-proton cross-section as a function of WIMP mass. The IceCube upper limit includes analysis of IceCube 22-string data (above $200 \mathrm{GeV}$ ) and preliminary analysis of AMANDA data (below $200 \mathrm{GeV}$ ).

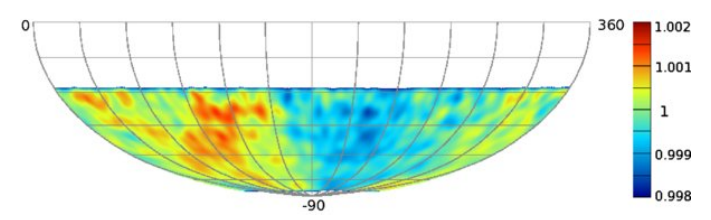

Figure 3: Fractional amplitude of the deviation from smooth isotropic distribution of cosmic ray arrival directions. In equatorial coordinates (note the right ascension coordinate is reversed compared to Fig. 1.)

configuration [3]. A large-scale excess and deficit at the level of 1-2 parts per thousand is observed. The overall location and orientation of these features is consistent with the large-scale anisotropy previously reported by Milagro [4] and ARGO [5] in the northern sky.

Acknowledgments: I am grateful for financial support from the Swedish Research Council (VR) through the Oskar Klein Centre.

\section{References}

[1] R. Abbasi et al. Time-Integrated Searches for Point-like Sources of Neutrinos with the 40String IceCube Detector. Submitted to Astrophys. J.

[2] R. Abbasi et al. Limits on a Muon Flux from Neutralino Annihilations in the Sun with the IceCube 22-String Detector. Phys. Rev. Lett., 102:201302, 2009.

[3] R. Abbasi et al. Measurement of the Anisotropy of Cosmic Ray Arrival Directions with IceCube. Astrophys. J., 718:L194, 2010.

[4] A. Abdo et al. The Large Scale Cosmic-Ray Anisotropy as Observed with Milagro. Astrophys. J., 698:2121.

[5] M. Amenomori et al. Anisotropy and Corotation of Galactic Cosmic Rays. Science, 314:439.

[6] J. Braun and D. Hubert. Proc. of the 31st ICRC, Lodz, Poland, 2009. arXiv:0906.1615. 